rheumatoid arthritis (RA). However, there is a dearth of knowledge in what role these transcripts play in fibroblast-like synoviocytes (FLSs) of RA patients. LncRNA GAPLINC, a novel long non-coding RNA, was first described in gastrointestinal cancer tissues and associated with bad behaviours of tumour cell as well as poor prognosis in patients

Objectives: This study was undertaken to explore the expression and roles of LncRNA GAPLINC in RA-FLSs and investigate its possible mechanism.

Methods: RA-FLSs and trauma-FLSs were cultured from synovial specimens. The expression of RNA was detected by qRT-PCR. GAPLINC suppression was transfected by siRNA. Cell viability analysis was taken by CCK-8 assay and flow cytometry. Cell invasion was using transwell chamber methodology. The bioinformatics analysis was performed using miRanda, PITA, RNAhybrid algorithms, as well as KEGG and Gene Ontology(GO) analysis.

Results: The relative expression of LnCRNA GAPLINC was significantly higher in RA-FLSs than trauma-FLSs $(p<0.05)$. Transfection of GAPLINC-siRNA significantly decreased the expression of LncRNA GAPLINC in RA-FLSs. GAPLINC suppression in RA-FLSs revealed significant alterations in cell proliferation and invasion. In the GAPLINC-siRNA group, a inhibition rate in growth was first observed (15.29\% $\pm 0.38 \%)$ at 24 hour after transfection, then a significant suppression was observed $(28.75 \% \pm 2.34 \%)$ at 48 hour, more apparent $(36.63 \%$ $\pm 7.93 \%)$ at 72 hour and largely maintained $(35.97 \% \pm 3.67 \%)$ at 96 hour after siRNA treatment, compared to the negative control group(NC-siRNA). Moreover, flow cytometry assay showed GAPIINC-siRNA group had an accumulation of cells in the G0/G1 phase and the decreased number of RA-FLSs in the S and G2/ $M$ phase. In the invasive assay, the membrane-invading RA-FLSs numbers decreased significantly in the treatment group with GAPLINC knockdown (45.0

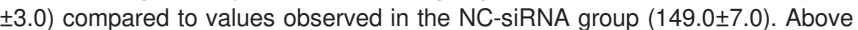
comparisons were all statistically significant $(p<0.05)$. The bioinformatics analysis predicted that some of microRNAs and mRNA may be the downstream molecules of LncRNA GAPLINC, we thus simulated a gene co-action network model based on the competitive endogenous RNA (ceRNA) hypothesis. Further verification of this model demonstrated that silencing of GAPLINC increased miR-382-5 $p$ and miR-575 expression.

Conclusions: The results suggest that elevated LncRNA GAPLINC expression promote the proliferation and invasion of RA-FLSs and it may function as a novel microRNAs sponging agent. Additionally, LncRNA GAPLINC may regulate RAFLS pathological behaviours in an miR-382-5 p-dependent and miR-575dependent manner. Based upon these findings, IncRNA GAPLINC may provide a novel valuable therapeutic target for RA patients.

Acknowledgements: This work was supported by grants from Province Natural Science Fund of Guangdong, China $\left.{ }^{\mathrm{No} .2014} \mathrm{~A} 030313080\right)$ and National Natural Science Foundation of China (No.81771750).

Disclosure of Interest: None declared

DOI: 10.1136/annrheumdis-2018-eular.3584

\section{THU0055 AN ANATOMICALLY DISTINCT PATHOGENIC FIBROBLAST SUBSET DRIVES INFLAMMATION IN ARTHRITIS}

A.P. Croft, J. campos, J. Marshall, J. Turner, F. Barone, A. Filer, C. Buckley. Rheumatology, University of Birmingham, Birmingham, UK

Background: Fibroblasts are key effector cells in the persistence of synovial inflammation and joint damage. It is not yet known whether specific subsets of synovial fibroblasts exist, and if so, if they are responsible for the distinct fibroblast mediated features observed in inflammatory arthritis, such as invasion of cartilage, bone damage, and persistence of inflammation.

Objectives: Here we identify and describe the biology of a functionally distinct pathogenic fibroblast cell type marked by the co-expression of Thy1.2 and Podoplanin (Pdpn), that is responsible for persistence of synovial inflammation.

Methods: We used the serum transfer arthritis (STA) model to induce joint inflammation. To identify putative subsets of fibroblasts we used flow cytometry using established markers of stromal cells, on enzymatically digested synovial tissue. These cells were localised in tissue sections using immunohistochemistry or immunofluorescence staining. Proliferation of subsets in vivo during the time course of STA was determined by BrdU incorporation studies. Transcriptomic analysis was performed using ultra-low input RNA sequencing on flow sorted populations of cells and single cell analysis performed on CD45 negative gated cells. Results: Using FAP as a biomarker of activated synovial fibroblasts we have been able to identify and define distinct subsets of synovial fibroblasts based on their co-expression of Thy 1.2 and Pdpn. These subsets reside in distinct compartments of the synovial microanatomy including the lining layer (LL), sub-lining layer (SL) and a subset of pericytes. We found that FAP +cells within the SL are highly proliferative and their expansion in cell number positively correlates with inflammation. Global deletion of FAP + mesenchymal cells in the synovium using a FAP-DTR mouse attenuated synovial inflammation; protected against erosive bone damage and lead to reduced leucocyte accumulation as a result of reduced chemokine and cytokine production by synovial fibroblasts within the membrane. Collectively these data suggest a pathogenic pro-inflammatory role for these cells.

Transcriptomic analysis by both ultra-low input bulk RNA sequencing and single cell analysis revealed these subsets to be transcriptionally distinct with the greatest transcriptional differences observed between LL and SL cells, demonstrating a site specific transcriptional program for cells within these compartments. Gene signature analysis of SL FAP +cells was consistent with an immune effector cell phenotype, in contrast to lining layer cells that express genes associated with matrix remodelling. Finally, to test this hypothesis we injected FAP + LL cells and $\mathrm{FAP}+\mathrm{SL}$ cells into inflamed ankle joints of mice. The injection of FAP $+\mathrm{SL}$ cells lead to more severe and prolonged joint inflammation, whereas injection of LL FAP + cells had no effect. Finally, we identified these cell subsets within human synovial tissue and have demonstrated the expansion of SL cells with inflammation.

Conclusions: Synovial inflammation is associated with the expansion, activation and differentiation of fibroblasts into distinct functional subsets of cells that regu late those specific aspects of inflammatory joint pathology. Direct targeting of spe cific pathogenic subsets of synovial fibroblasts may provide a novel, nonimmunosuppressive approach to the treatment of inflammatory arthritis.

Disclosure of Interest: None declared

DOI: 10.1136/annrheumdis-2018-eular.7151

\section{THU0056 \\ 14-3-3 IS A MOLECULAR SWITCH REGULATING MACROPHAGE POLARISATION IN INFLAMMATORY ARTHRITIS}

W. Fu, A. Hettinghouse, C. Liu. Department of Orthopaedic Surgery, New York University Medical Center, New York, USA

Background: Functional heterogeneity is a hallmark of macrophages, which can classified into 2 major phenotypes with opposite role in inflammation termed M1 (inflammatory or classically activated) macrophages and M2 (alternatively activated) macrophages. In addition, M1-M2 polarisation of macrophages is a highly dynamic process and the phenotype of polarised macrophages can be switched under physiological and pathological conditions. Progranulin (PGRN), a multiple functional growth factor, binds to TNF receptor 2 (TNFR2) and activates the protective and anti-inflammatory pathway in inflammatory arthritis. In addition, 14-33e was identified as a component of PGRN/TNFR2 complexes in Raw264.7 macrophages.

Objectives: In this study, we examined whether 14-3-3e regulated macrophage polarisation and if so, whether this was important for PGRN's anti-inflammatory action in inflammatory arthritis.

Methods: LysMCre and14-3-3 $\epsilon$ F/F mouse line was obtained from Jackson Laboratory.

Results: : 14-3-3e regulates macrophage polarisation in vitro. We found that 14-3-3e deficiency enhanced M1 while inhibited M2 polarisation (figure 1a, b). Interestingly, PGRN showed reverse effects on macrophage polarisation. In addition, PGRN's effects were largely lost in 14-3-3e deficient BMDMs (figure 1a, b). Together, these data indicate that $14-3-3 e$ is a critical downstream mediator of PGRN regulation of macrophage polarisation.

Macrophage-specific 14-3-3e contributes to control of inflammatory arthritis and is critical for PGRN's anti-inflammatory action. We then explored the role of macrophage-specific $14-3-3 e$ in inflammatory arthritis and whether PGRN's anti-inflammatory activity depended on $14-3-3 e$ in vivo. We established $\mathrm{CIA}$ in $14-3-3 e F / F$ (serve as WT) and $14-3-3 e \Delta / \Delta$ mice, followed by i.p. injection of recombinant PGRN. The clinical arthritis score demonstrated that $14-3-3 e \Delta / \Delta$ mice displayed increased severity of CIA compared with WT CIA. In addition, PGRN's protective effects against inflammatory arthritis was compromised in 14 $3-3 e \Delta / \Delta$ mice (figure 1c), suggesting that $14-3-3 e$ is critical downstream mediator of PGRN's anti-inflammatory effects. In addition, FACS analysis showed that total numbers of F4/80 cells were not different in all WT and knockout CIA mice However, analysis of CD45 +CD11b+cell population in spleen demonstrated a significant increase in mean fluorescence intensity (MFI) of iNOS and a significant decrease of CD206 +cells in PBS treated 14-3-3e $\Delta / \Delta$ CIA mice as compared with PBS treated WT CIA mice; moreover, there was a significant decrease of iNOS MFI while a dramatic increase of CD206 +cells in PGRN-treated WT mice compared to that in PBS-treated mice. Further PGRN-mediated effects on macrophage polarisation was lost in $14-3-3 e \Delta / \Delta$ CIA mice (Fig 1d, e). Cellectively, these results indicate that PGRN skews macrophage toward M2 polarisation to resolve inflammation and this effect depends on $14-3-3 \epsilon$. 


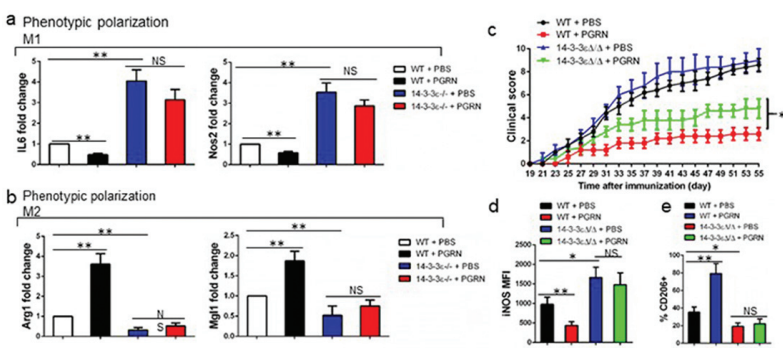

Abstract THU0056 - Figure 1. 14-3-3e is a critical mediator of PGRN' regulation of macrophage polarisation and contributes to PGRN's anti-inflammation action. (a, b) qPCR analysis of II1b and Nos2 (a), or Arg1 and Mgl1 (b) mRNA expression in WT, or 14-3-3e-/macrophages which are polarised to M1 (a) or M2 (b) in the the absence or presence of PGRN (200ng/ml). (c) Clinical arthritis score of WT or 14-3-3 $\epsilon \Delta / \Delta$ CIA mice treated with or without PGRN. $n=8$ (d, e) CD45+CD11b+ cells were analysed for MFL of iNOS (d) and percentage of CD206+ cells (e). ${ }^{*} \mathrm{p}<0.05,{ }^{* \star} \mathrm{p}<0.01, \mathrm{NS}=$ no significance

Conclusions: Both in vitro and in vivo results indicate that $14-3-3 \epsilon$ is a key molecule regulating macrophage polarisation which plays an important role in inflammatory arthritis, and it is an essential component for PGRN/TNFR2 mediated protective effect against inflammatory arthritis.

Disclosure of Interest: None declared

DOI: 10.1136/annrheumdis-2018-eular.4729

\section{THU0057 MECHANICAL STRAIN DETERMINES THE SITE- SPECIFIC DIRECTION OF INFLAMMATION AND TISSUE DAMAGE IN ARTHRITIS}

I. Cambré ${ }^{1}$, D. Gaublomme ${ }^{1}$, A. Burssens ${ }^{1}$, P. Jacques ${ }^{2}$, L. Meuris ${ }^{3}$ S. Lambrecht ${ }^{2}$, S. Carter ${ }^{4}$, A. De Muynck ${ }^{5}$, N. Callewaert ${ }^{3}$, G. Kollias ${ }^{6}$, R. Lories ${ }^{4}$ G. Schett ${ }^{7}$, D. Elewaut ${ }^{1} .{ }^{1}$ Rheumatology, UGENT/VIB; ${ }^{2}$ Rheumatology, UZ Gent, ${ }^{3}$ Biochemestry and Microbiology, UGENT/VIB, Ghent, ${ }^{4}$ Development and Regeneration, KUL, Leuven; ${ }^{5}$ Physics and astronomy, UGent, ${ }^{6}$ Experimental Physiology, Biomedical Sciences Research Center 'Alexander Fleming'; ${ }^{7}$ Rheumatology and Immunology, Friedrich Alexander University of ErlangenNuremberg and Universitatsklinikum, Ghent, Belgium

Background: Many pro-inflammatory pathways leading to arthritis act systemically on the immune system rather than locally in the joint. However, the reason behind the regional and patchy distribution of arthritis represents a longstanding paradox.

Objectives: To explore the relation between mechanical strain and joint inflammation and to understand the underlying basis of joint pattern involvement in inflammatory rheumatic diseases.

Methods: Arthritis was induced by collagen-induced arthritis $(\mathrm{CIA})$ and passive collagen antibody induced arthritis (CAIA) in respectively C57BL/6 and RAG2-/( $T$ - and B-cell deficient) mice. Animals were subjected to different regimens of mechanical strain. Increased strain occurred in voluntary running mice whereas tail suspension (unloading) abolished mechanical strain; both were compared to control housing conditions. The impact of different loading conditions was measured on clinical disease score, histology, micro-CT images and erosion quantification, gene induction in tendon and synovial tissue, immune cell recruitment in situ, development of anti-collagen antibodies and their pattern of siaylation and galactosylation.

Results: Voluntary running of CIA in C57BL/6 mice markedly induced an early onset and increased progression whereas no disease onset could be observed in the hind paws from animals in unloaded conditions. CAIA in running RAG2-/- mice also induced early arthritic symptoms and severe progression. Intriguingly, running conditions were sufficient to induce arthritis without the need of LPS as an inflammatory trigger. Mechanical strain did not alter however IgG autoantibody levels nor their levels of galactosylation and sialylation. Furthermore, we demonstrate that mechanical strain on stromal cells results in recruitment of classical monocytes into specialised mechano-sensitive regions characterised by a unique microanatomy. This promotes local inflammation and differentiation into local osteoclasts which induce regional erosions. A striking similarity was observed in the pattern of joint erosions in human patients with RA and SpA which were also confined to these mechanosensitive regions.

Conclusions: This study provides the first evidence that mechanical strain controls the transition from systemic autoimmunity into site-specific joint inflammation.
Homing of inflammation and development of erosions was confined to specific mechano-sensitive regions, characterised by a high number of attachment- and contact points for tendons. This represents a novel paradigm and explains why arthritis in mice and humans is characterised by a regional and patchy distribution. Curiously, this pathway does not rely on adaptive immunity but rather on stroma cells. Mechano-stimulation of mesenchymal cells induced CXCL1 and CCL2 permitting recruitment of classical monocytes which can differentiate into boneresorbing osteoclasts. Thus, mechanical strain controls the site-specific direction of inflammation and tissue damage in arthritis.

Acknowledgements:

Disclosure of Interest: None declared

DOI: 10.1136/annrheumdis-2018-eular.4736

\section{THU0058 TAS8274, A HIGHLY SELECTIVE JANUS KINASE 3 INHIBITOR, SHOWS POTENT EFFICACY, BUT DOES NOT AFFECT HOST DEFENSE, IN PRECLINICAL MOUSE MODELS}

H. Hayashi, S. Demizu, K. Tanaka, M. Mitsuya, A. Saito, T. Utsugi, E. Sasaki, Y. Iwasawa. Taiho Pharmaceutical co., LTD., Tsukuba, Ibaraki, Japan

Background: The family of Janus kinases (JAKs) plays important roles in signalling pathway mediated by various cytokine receptors. An aberrant activation of JAK-STAT signalling has been reported to be involved in the pathogenesis of autoimmune diseases ${ }^{1}$. Pan-JAK inhibitors have shown a good efficacy in patient with rheumatoid arthritis $(R A)^{2}$. However, their use is limited due to safety concerns, including severe herpes zoster infection, by inhibiting JAK1-mediated interferon signaling ${ }^{3}$. Therefore, a selective JAK3 inhibitor would provide a better balance between efficacy and safety than pan-JAK inhibitors.

Objectives: We identified the characteristics of TAS8274, a novel highly selective inhibitor of JAK3, using in vitro assays, a mouse model of collagen-induced arthritis (CIA), and a mouse model of herpes simplex virus (HSV)-1 infection.

Methods : In vitro biochemical assay was performed using available kinase assay panels. The effects on anti-inflammatory responses were assessed by examining cytokine productions. IL-2, IL-3, and IFN- $\alpha$-induced phosphorylation of STAT proteins in peripheral blood mononuclear cells (PBMCs) were analysed by a flow cytometry method. NK cell cytotoxicity in the presence of IFN- $\alpha$ was evaluated by $\mathrm{Cr}^{51}$ release assay. In a mouse skin HSV-1 infection model, TAS8274 and tacrolimus were administered for 7 days before inoculation of the virus on the back skin, and then were administered for another ten consecutive days. At the end of this experiment, the number of papules on the back was counted. To evaluate the therapeutic efficacy using mouse CIA model, TAS8274 was orally administered to CIA mice after the disease onset. Disease severity was evaluated by clinical score of paw swelling, and the scores of inflammation, pannus, cartilage, and bone damage were performed using a modified Mankin score system.

Results: TAS8274 inhibited the enzymatic activity of JAK3 $\left(\mathrm{IC}_{50}=0.16 \mathrm{nM}\right)$, and showed more than 1000-fold selectivity against other JAK kinases. In the cellbased assays, TAS8274 strongly inhibited IL-17 production from differentiated Th17 cells. TAS8274 also suppressed the IL-2-induced STAT5 phosphorylation in PBMCs, but had much lower inhibitory effects on the IFN- $\alpha$-induced STAT1 phosphorylation. In contrast, Tofacitinib and Baricitinib had robust inhibitory effects on the IFN- $\alpha$-induced STAT1 phosphorylation. Furthermore, Tofacitinib and Baricitinib dose-dependently reduced the NK cell cytotoxicity, while TAS 8274 had little effect on that. Tacrolimus-treated group significantly increased the number of papules compared with vehicle-treated group in a mouse HSV-1 infection model, but TAS8274-treated group did not increase the number of papules. In an established mouse CIA model, TAS8274 dose-dependently reduced the severity of arthritis and histopathological scores compared with vehicle-treated mice. Conclusions: TAS8274 did not inhibit the JAK3-independent STAT signalling pathway in vitro and showed potent efficacy at dose range without exacerbation of the risk of HSV-1 infection. Our study demonstrates that TAS8274 would be an attractive therapeutic agent with excellent balance between efficacy and safety.

\section{REFERENCES}

[1] Immunity 2012;36,542-50.

[2] Ann Rheum Dis. 2013;72,111-5.

[3] Arthritis Rheumatol 2014;66:2675-84.

Disclosure of Interest: None declared DOI: 10.1136/annrheumdis-2018-eular.1865 